\title{
LE BOIS DE LUTHERIE
}

\author{
V. BUCUR \\ Institut National de la Recherche Agronomique, Centre de Recherches Forestières de Nancy, \\ F-54280 Champenoux, France
}

\begin{abstract}
It is an understatement to say that wood is an unique material used in the art of musical craftmanship. Under the generic name of "resonance wood" are admitted all species used for musical instruments. In this note our comments are limited to spruce and curly maple for violins. The violin makers traditionally select their boards according to the simplest macroscopic criteria as straight grain, fine structure and light density. The description of some acoustical parameters for the characterization of wood for violins was first made on small thin specimens using resonance frequency method. Here we present nine acoustical parameters determined with ultrasonic velocity method. Factors affecting acoustical and physical properties of resonance wood are discussed. We refere to the natural ageing of wood and to the behaviour of this material under long term loading.
\end{abstract}




\section{Introduction.}

La dénomination de "bois de lutherie" ou "bois de résonance" est donnée aux essences ligneuses utilisées pour la facture instrumentale.

Pour comprendre le mécanisme de l'émission sonore d'un instrument de musique à cordes, il est indispensable de se préoccuper, d'une part de la physique de l'émission sonore, et d'autre part de la qualité du matériau dont la caisse de résonance de l'instrument est fabriqué, c'est-à-dire le bois.

Par ailleurs, il est intéressant de noter que, pendant les deux dernières décennies, une vraie acoustique des instruments de musique $s$ 'est développée $[1,2,3]$.

Dans ce qui suit, nous essaierons de donner un aperçu sur les propriétés physiques des essences ligneuses les plus utilisées dans la facture des instruments à cordes. Pour illustrer nos propos, nous avons choisi l'épicéa pour la table d'harmonie et l'érable ondé pour le fond des instruments à archet. Le but de cette note est de relier l'art du luthier à l'acoustique et à la science du bois en montrant qu'il est possible de s'appuyer sur les propriétés physiques du bois, objectivement mesurables,, pour obtenir pour chaque instrument les qualités acoustiques recherchées.

D'après l'opinion des musiciens, les violons doivent présenter un certain nombre de qualités qui sont fonction de constantes acoustiques du bois de résonance utilisé. Ainsi par exemple, la clarté du jeu du violon pourrait s'exprimer par la vitesse de propagation du son et le phénomène de l'extinction du son par 1 'amortissement ou $1^{\prime}$ atténuation des vibrations dans le bois.

Pour choisir le bois de résonance d'épicéa pour la table du violon, les luthiers font usage des critères empiriques suivants : matériau totalement dépourvu d'imperfections anatomiques, accroissements de largeur 0,8 à $2,5 \mathrm{~mm} d^{\prime}$ une régularité presque parfaite, longues périodes de séchage naturel, masse volumique relativement faible $\left(400 \mathrm{~kg} / \mathrm{m}^{3}\right)$. Pour le bois d'érable, le critère de sélection est 1 'aspect, car les planches présentant une ondulation du fil du bois très marquée sont les plus recherchées en raison du bel effet esthétique qu'elle donne après vernissage. (Des détails sur la structure du bois de résonance des violons de notre siècle sont donnés dans [4].

L'objectif de nos recherches a été de développer des méthodes non destructives capables d'élucider les relations existant entre différents paramètres physiques du bois de résonance pour caractériser son comportement élastique.

Historiquement, les premières méthodes dynamiques employées pour la mesure de modules d'élasticité et de l'amortissement, dans la direction longitudinale ou radiale des échantillons de bois ont été celles de la fréquence de résonance $[5,6,7]$. Les méthodes ultrasonores développées plus tard grâce à l'évolution de la technologie des transducteurs ont permis $l^{\prime}$ accès à toutes les constantes de rigidité du bois considéré comme un solide orthotrope 


\section{Considération théoriques}

La loi de propagation des ondes élastiques dans les solides orthotropes permet d'établir les relations entre les vitesses de propagation des ultrasons et les constantes de rigidité, selon 1 'équation de CHRISTOFFEL :

$\left(C_{i j k l} n_{i} n_{j}-\rho v^{2} \delta_{i k}\right)=0$.

où : $C_{i j k l}$ sont les rigidités, $n_{j}$ et $n_{j}$ les cosinus directeurs du vecteur de propagation, o la densité, $V^{j}$ la vitesse de propagation de l'onde, $\delta_{i k}$ le tenseur de Kronecker.

Les solutions de cette équation montrent qu'il est possible d'associer à chaque axe d'anisotropie du solide des vitesses caractéristiques de la propagation des ondes longitudinales et transversales ainsi que les rigidités correspondantes. En notation conventionnelle matricielle, les composantes diagonales du tenseur de rigidité $C_{i j k l}$ sont $C_{11} \ldots C_{66}$ dans la base naturelle orthonormée $(1,2,3$ ou $L, R, T$ qui sont les axes correspondant aux directions principales de croissance de 1 'arbre). Les vitesses respectives seront notées $v_{11} \ldots v_{66}$.

L'anisotropie du bois peut être exprimée par les rapports des vitesses dans les trois axes ou par les rapports des invariants acoustiques. Ainsi par exemple, 1 'expression de l'invariant acoustique correspondant au plan 1,2 est la suivante :

$I_{12}=\left[\left(v_{11}^{2}+v_{22}{ }^{2}+2 v_{66}{ }^{2}\right)\right]^{1 / 2}$

Par permutations, nous obtenons $l^{\prime}$ expression des autres invariants correspondant aux plans 1,3 et 2,3 . Le rapport des invariants a été calculé selon la relation :

$I_{\text {ratio }}=I_{23} / 0.5\left(I_{12}+I_{13}\right)$

Ce rapport considère les valeurs des vitesses dans le plan transversal $(2,3)$ et la moyenne des vitesses dans les plans $(1,2$ et $1,3)$ contenant $I^{\prime}$ axe longitudinal.

\section{Constantes élastiques des bois de résonance.}

Pour déterminer les constantes élastiques du bois de résonance, nous avons choisi les variétés d'épicéa et d'érable de résonance les plus employées par les luthiers du monde entier. Les vitesses de propagation des ultrasons et les constantes élastiques correspondantes sont données dans les Tableaux 1 et 2 . Comme on ' $\mathbf{s}^{\prime} \mathbf{y}$ attendait, les valeurs des vitesses selon $l^{\prime a x e}$ longitudinal sont les plus élevées. Les valeurs les plus faibles observées correspondent aux vitesses des ondes de cisaillement se propageant dans le plan transversal du bois.

Le tableau des termes de la matrice des rigidités montre des valeurs très élevées pour le module longitudinal et des valeurs faibles pour le module de cisaillement dans le plan transversal.

L'anisotropie, exprimée par le rapport des vitesses, donne un coefficient maximum de 4.5 , tandis que le rapport des invariants $(0,45$ pour l'épicéa et 0,63 pour l'érable ondé) illustre la forte 
anisotropie de l'épicéa. Considérant ce paramètre, nous pouvons remarquer que l'anisotropie de l'érable ondé, si différente de celle de l'épicéa, joue un certain rôle dans l'acoustique des instruments et que son choix empirique par les luthiers n'a pas simplement une motivation esthétique.

Les constantes calculées selon l'équation (1) nous donnent la réponse globale du bois à l'échelle macroscopique. Selon cette équation, la dispersion spatiale de la vitesse de phase n'est pas prise en compte. Lorsque la longueur $d^{\prime}$ 'onde est supérieure aux dimensions de la structure anatomique du matériau, la réponse élastique est correctement représentée.

Cependant, il peut être intéressant de s'interroger sur l'influence que peut avoir sur les propriétés macroscopiques la nature discrète de la structure du bois. Ceci nous amène à nous intéresser à la réponse élastique locale du bois, car l'inhomogénéité à l'échelle microscopique peut engendrer des effets observables à l'échelle macroscopique.

Les données présentées dans le Tableau 3 visent à mettre en évidence le comportement local du bois de résonance, à cernes annuels relativement larges, pour les zones de bois final et initial. Les caractéristiques microdensitométriques ont été relevées sur barrettes ainsi que les vitesses des ondes longitudinales dans les directions $L$ et $T$. La zone explorée par ultrasons a été limitée par les dimensions de la surface de contact du transducteur $\left(1 \mathrm{~mm}^{2}\right)$. Les valeurs très faibles des vitesses V11 sont probablement dues à la dispersion géométrique du faisceau ultrasonore. Les barrettes de faible section $\left(5 \times 3 \mathrm{~mm}^{2}\right)$ sont susceptibles d'engendrer des phénomènes de diffraction ou de diffusion. A celà viennent s'ajouter la dispersion viscoélastique et la possibilité d'apparition d'ondes de stonley. Il est généralement admis que ces phénomènes sont négligeables lorsque la longueur $d$ 'onde est 4 fois supérieure à la taille des fibres. (Evidemment, ce n'était pas notre cas). Auparavant, il faudrait $\mathbf{s}^{\prime}$ assurer aussi que la condition de "milieu infini" est respectée et que les couches de bois final ne jouent pas le rôle de guide d'onde pour favoriser les ondes les plus rapides.

Pour améliorer la précision de la mesure ultrasonore à l'échelle du cerne annuel, il faudrait suivre la voie ouverte par la microscopie acoustique et par la spectroscopie ultrasonore.

\section{L'ancienneté du bois.}

L'ancienneté du bois s'exprime habituellement par le temps de vieillissement naturel du matériau après l'abattage de l'arbre.

Pour le luthier, le terme a un sens un peu plus large, car à la suite des sollicitations mécaniques ou thermo-hydriques répétées, induites par le jeu de l'instrument, le bois est capable de modifier ses propriétés. Il existe un parallélisme étonnant entre ces modifications et celles engendrées par le vieillissement naturel du matériau, mais il reste à savoir si les modifications ainsi engendrées se maintiennent quel que soit le temps d'usage d'un instrument musical. 
Pour le bois de résonance, les luthiers, les musiciens, les acousticiens, les forestiers ont recommandé différentes périodes de séchage naturel. Pendant des périodes courtes de séchage, de 3 à 10 ans, il s'agit d'une stabilisation dimensionnelle et de forme du bois attribuée à la fois à l'équilibre hygroscopique du matériau et à la stabilisation de toutes les tension internes. Les luthiers ont observé, sans l'expliquer, le phénomène de "durcissement" du bois suite au vieillissement du matériau. Dans notre cas il s'agit d'un processus évolutif qu'on peut attribuer à des modifications dans la structure cristallographique de la cellulose, qui peut générer des modifications de la valeur de l'angle des microfibrilles. Ce phénomène a été mis en évidence par la méthode de diffraction aux rayons $\mathrm{X}$.

$D$ 'autre part, les longues périodes de séchage ne doivent pas dépasser 100 ans, car après, le bois devient friable et ses propriétés acoustiques et mécaniques s'amoindrissent.

Il est courant de dire que les instruments anciens sont bons parce qu'ils sont vieux. On dit aussi qu'il faut beaucoup jouer d'un instrument neuf pour le rendre bon, qu'il faut le "faire". D'autre part, il est bien vrai qu'avec l'âge, un mauvais instrument ne s'améliore pas. Il semble incontestable que le jeu du violon génère des sollicitations de longue durée dans le bois qui ont une influence observable sur la qualité sonore du violon.

Ce processus peut être simulé sur éprouvettes par des sollicitations de longue durée reproduisant d'une façon schématique les sollicitations engendrées dans le bois du violon par l'utilisateur de l'instrument.

Selon leur nature, nous pouvons distinguer deux groupes importants de sollicitations de longue durée qui agissent dans le bois du violon : des sollicitations mécaniques et des sollicitations thermohydriques.

Les modifications des vitesses de propagation des ultrasons dans les bois, produites à la suite des sollicitations de longue durée de flexion statique et dynamique dans le domaine de faible contrainte (0.2 $\sigma$ Rupture) sur des éprouvettes d'épicéa, de sapin et d'érable ondé, ont été rapportées par [8].

Des modifications importantes des vitesses de propagation sont observées au commencement du processus de simulation, maís après une certaine durée, variable selon la nature de la charge (10...12 jours), une nette stabilisation apparait. Dans le cas du bois de lutherie, il est probable qu' à un certain moment, le phénomène de fatigue, dû au jeu du violon, devient stationnaire.

Il apparaît que la direction de l'anisotropie selon laquelle la charge a été appliquée est déterminante pour les modifications de la structure. Si la sollicitation de flexion statique suit la direction longitudinale, toutes les valeurs de la vitesse dans les trois directions sont diminuées de 0.6 à $6.6 \%$.Il convient d'insister ici sur la fluctuation du timbre des violons neufs. Cette fluctuation est probablement dépendante des modifications des vitesses de propagation des ondes dans le bois de la caisse de résonance de l'instrument qui, à leur tour, sont déterminées par les modifications de la structure fine du matériau concernant peut-être l'angle des microfibrilles et les liaisons $\mathrm{OH}$ des hémicelluloses. 


\section{Conclusions.}

Lorsqu'il s'agit de la détermination des constantes élastiques des bois de résonance dans toutes les directions d'anisotropie, sur des échantillons de relativement petite taille, les ultrasons constituent un outil extrêmement souple et efficace qui pourrait être facilement mis à la disposition des luthiers.

Par son côté prospectif, ce travail sur le bois de résonance a mis en lumière un certain nombre de questions qui pourraient susciter des études ultérieures.

Enfin, si ce travail avait permis d'illustrer la multitude des problèmes que le bois de résonance en particulier, et le bois massif en général, peuvent poser à l'acousticien, ses ambitions auraient été atteintes.

\section{Bibliographie.}

[1] BENADE, A.H. (1976) : Fundamentals of musical acoustics. Oxford University Press, New York.

[2] HUTChINS, C.M. (1962) : Benchmark papers in acoustics. Part I: Violin family components. Part II: Violin family functions. Hutchinson \& Ross Inc. Dowen, USA, - Academic Press Inc.

[3] CREMER, L. (1984) : The physics of the violin. MIT, Cambridge, MA, USA.

[4] ROCABOY, F., BUCUR, V. (1990) : About the physical properties of wood of twentieth century. CATGUT ACOUSTICAL SOCIETY J. 1(6) serie II, 21-28.

[5] BARDUCCI, I, PASQUALINI, G. (1948) : Misura dell'attrito interno e delle costanti elastiche del legno. NUOVo CIMENTO I(5) 416-466.

[6] HOLZ, D. (1967) : Untersuchungen an Resonanzholz. HOLZTECHNOLOGIE, Part 3- 8(4) 221-224, Part 4- 9(4) 225-229, 1968, Part 5- 14(4) 195-202, 1973.

[7] HAINES, D.(1979) : On musical instrument wood. CATGUT ACOUST. SOC. NEWSLETTER, $\mathrm{n}^{\circ} 31,23-32$.

[8] BUCUR, V., GHELMEZIU, N.(1977) : The influence of long term loading on acoustic properties of resonance woods. INDUSTRIA LEMNULUI 28(4), 171-180.

[9] BUCUR, V., (1987) : Varieties of resonance wood and their elastic constants. J. CATGUT ACOUST. SOC 47, May, 42-48. 
Table 1 : Vitesses de propagation des ultrasons à 1 MHz mesurées sur échantillons d'épicéa et d'érable de résonance [9]

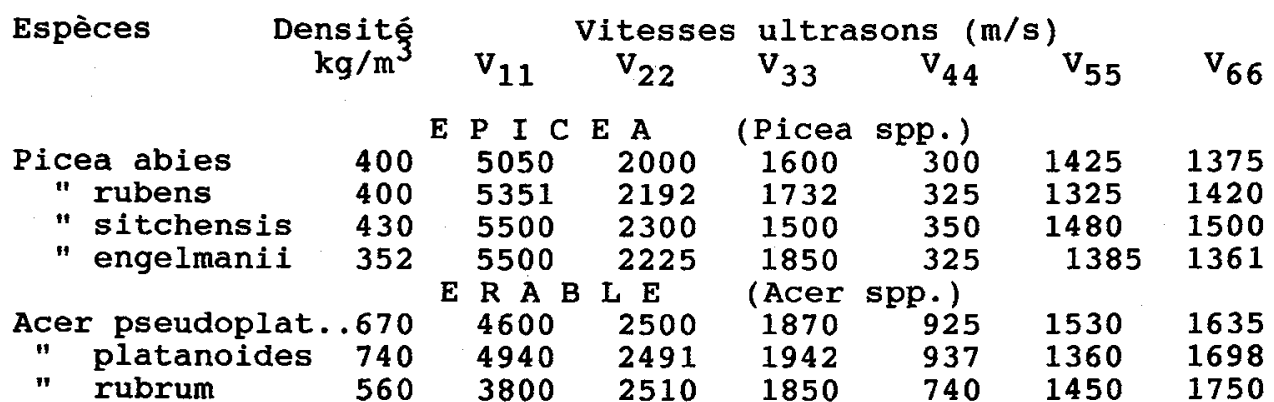

Table 2 : Termes de la matrice des rigidités du bois de résonance calculées à partir des mesures ultrasonores [9]

Espèces

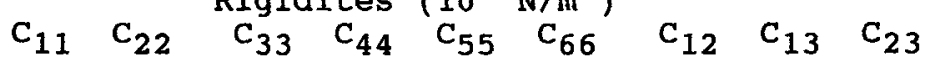

P.abies $\quad 102 \quad 16$ P I C E A (Picea spp.)

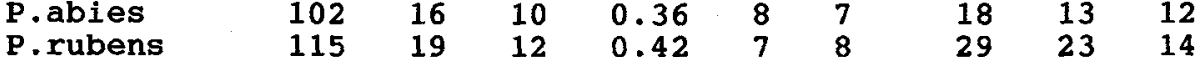



$\begin{array}{llllllllll}\text { P.engelmanii } & 106 & 17 & 12 & 0.37 & 7 & 7 & 27 & 21 & 14\end{array}$

\begin{tabular}{lrrrrrrrrr} 
& & E & R A B & L E & \multicolumn{7}{c}{ (ACer spp.) } & & \\
A.pseudoplat & 141 & 42 & 23 & 5.73 & 15 & 23 & 32 & 31 & 19 \\
A.platanoides & 180 & 46 & 28 & 7.20 & 14 & 21 & 54 & 44 & 16 \\
A.rubrum & 81 & 35 & 19 & 3.06 & 12 & 18 & 12 & 14 & 13
\end{tabular}

Table 3 : Vitesses de propagation des ultrasons et composantes microdensitométriques du bois initial et final d'épicéa et de sapin de résonance.

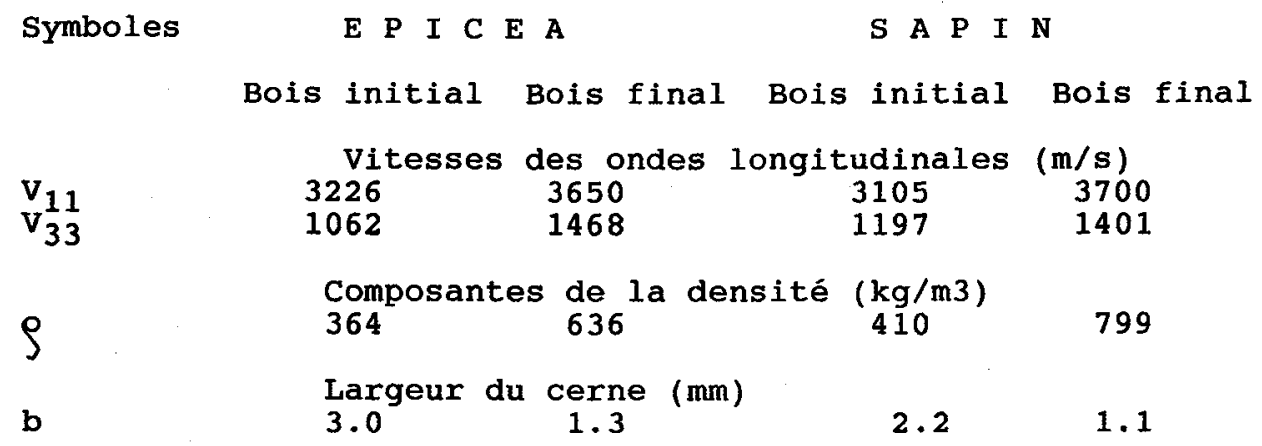

